\title{
Mechanics of tungsten blistering II: Analytical treatment and fracture mechanical assessment
}

\author{
Muyuan $\mathrm{Li}^{\mathrm{a}}$, Jeong-Ha You ${ }^{\mathrm{a}, *}$ \\ ${ }^{a}$ Max-Planck-Institut für Plasmaphysik, Boltzmannstr.2, 85748 Garching, Germany
}

\begin{abstract}
Since a decade the blistering of pure tungsten under hydrogen implantation has been one of the major research topics in relation to the plasma-wall interaction of tungsten-armored first wall. Overall blistering may reduce the erosion lifetime of the wall. Mature blisters grown by high internal pressure are likely to burst leading to exfoliation of the surface. Therefore, the control and suppression of blistering is an important concern for sustainable operation of the tungsten-armored plasma-facing components. In this context, a quantitative assessment of the mechanical conditions for blister bulging and growth is an important concern.

In this article we present a theoretical framework to describe the bulging deformation of tungsten blisters and to estimate the mechanical driving force of blister growth. The validity of the analytical formulations based on the theory of elastic plates is evaluated with the help of finite element analysis. Plastic strains and $J$-integral values at the blister boundary edge are assessed by means of numerical simulation. Extensive parametric studies were performed for a range of blister geometry (cap aspect ratio), gas pressure, yield stress and hardening rate. The characteristic features of the blistering mechanics are discussed and the cracking energy is quantitatively estimated for the various combinations of parameters.
\end{abstract}

Keywords: tungsten, plasma-facing armor, blister, crack, $J$-integral, strain energy release rate

\section{Introductions}

Blistering is an irreversible surface damage phenomenon of a solid occurring often under hydrogen or helium ion bombardment and characterized by the formation of microscopic blisters filled with high-pressure gas [1]. Since a decade the blistering of pure tungsten under hydrogen implantation has been one of the major research topics in the plasma-wall interaction community in relation to the physical compatibility of tungsten-armored first wall with fusion plasma.

Depending on the fluence of incident hydrogen and surface temperature, significant blistering may develop on the surface of tungsten [2, 3]. Since the solubility of hydrogen in tungsten is extremely low, the excessive

\footnotetext{
*Corresponding author. Tel.: +49 (0)89 3299 1373; fax:+49 (0)89 32991212 .

Email address: you@ipp.mpg.de (Jeong-Ha You)
} 
hydrogen solutes are populated beneath the surface and readily trapped at crystal defects, where the solutes precipitate as tiny gas bubbles and subsequently agglomerate together forming a blister of larger size ranging between one and hundreds of $\mu \mathrm{m}[\underline{3}$ ]. Fig. 1 shows a typical blister on tungsten [1]. Blisters can accommodate a considerable amount of fuel gas thus raising the issue of tritium retention [4].

Overall blistering may reduce the erosion lifetime of the wall. Mature blisters grown by high internal pressure are likely to burst leading to exfoliation of the surface [5]. The loss of material by such exfoliation could be far more significant than that of physical sputtering that is very small for tungsten. Therefore, the control and suppression of blistering is an important concern for sustainable operation of plasma-facing components. In this context, quantitative assessment of the mechanical conditions for blister growth is as important as the understanding of physical mechanism. The knowledge on the impact of blister geometry, pressure and material properties on the driving force of blister growth would provide deeper insight into the blistering behavior.

In literature, there are several mechanics-based studies on the deformation and growth of a (already formed) blister. These works can be categorized into analytical [6, 7, 8] and numerical [9, 10] studies. The analytical studies were mostly focused on adapting the closed elastic stress solutions of a pressurized thin plate to a blister cap on the basis of plate theories and studying blister growth which was modeled as a crack extension event in the framework of linear elastic fracture mechanics. The numerical studies aimed at quantitative computation of stress and strain fields of a three-dimensional blister system for various loading parameters and boundary conditions by means of finite element analysis (FEA).

In this paper, we present a comparative study of blister mechanics where the deformation and the growth of a tungsten blister are modeled and compared on the basis of plate theories, analytical linear elastic fracture mechanics (LEFM) and FEA-based LEFM and elasto-plastic fracture mechanics (EPFM) simulations, respectively. The validity of the analytical models is discussed with reference to the computational prediction. Emphasis is placed on rigorous and systematic evaluation of the methodologies. In addition, the results of parametric investigation are presented for a range of yield stress, size, thickness and hardening behavior of the tungsten blister.

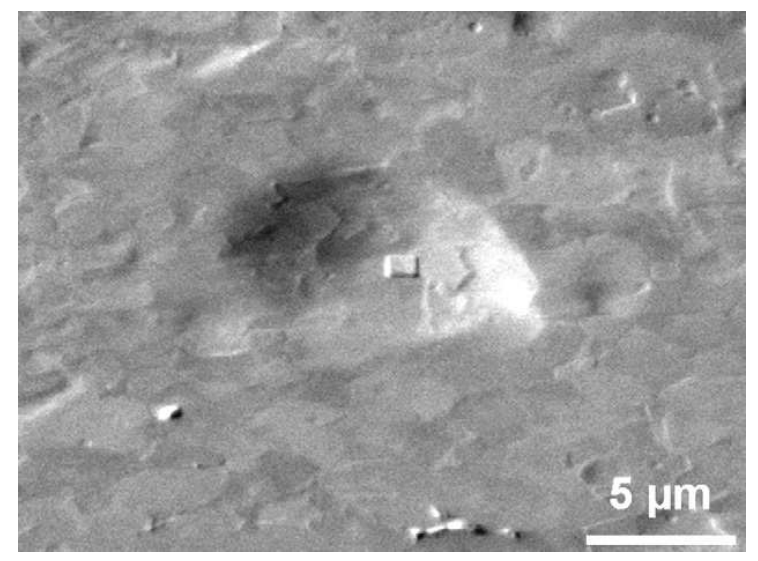

Fig. 1. Scanning electron micrograph of a typical blister on a tungsten sample irradiated by deuterium ions [1]. 


\section{Theoretical background for analytical models}

\section{Thin plate}

In the analytical study of blistering mechanics, the theory of elastic thin plates is often applied (e.g. [7, 8] ). In this approach, a blister cap is modeled as a thin circular plate where its edge section is assumed to be fully restrained along the periphery by cantilever boundary condition. In Fig. 2, an axi-symmetric plane model of a pressurized blister is schematically illustrated. Uniform gas pressure is applied onto the lower (i.e. inner) face of the plate to produce the deformation of the cap.

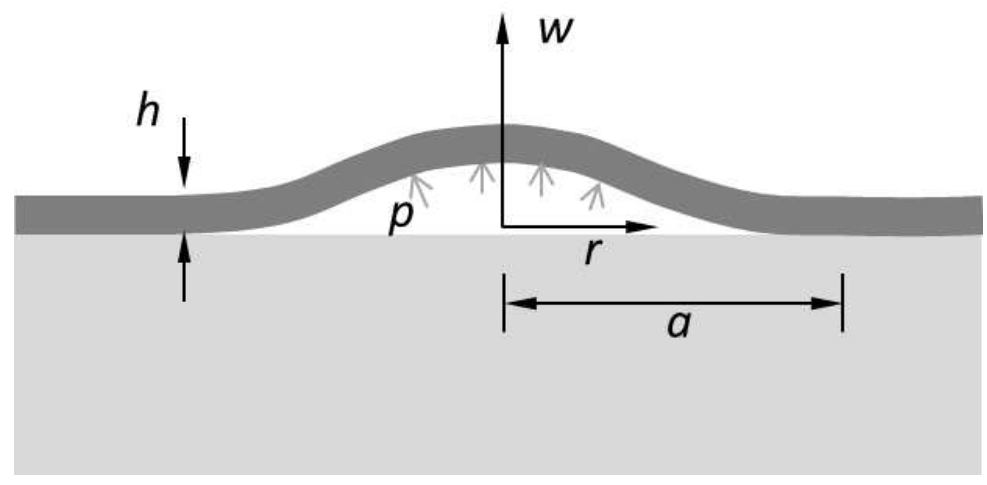

Fig. 2. A schematic drawing blister model based the assumption of thin plate. $p$ is the gas pressure in the blister, $a$ is the blister radius and $h$ is the thickness of the blister cap.

The analytical solution derived by Timoshenko can be used for pressurized thin circular plates [11]. In the following Timoshenko's thin plate theory is briefly summarized.

The plate is assumed to be thin and the vertical deflection is small compared to the thickness of the plate. When the edge is fully clamped, the slope of the vertical deflection must be zero at $r=0$ and $r=$ $a$. At the edge of the plate $(r=a)$, the deflection is zero as well. Applying these boundary conditions, the vertical deflection, $w$, is given by

$$
w=\frac{p}{64 D}\left(a^{2}-r^{2}\right)^{2}
$$

where $D=\frac{E h^{3}}{12\left(1-\nu^{2}\right)}$ is bending stiffness, $E$ is the Young's modulus and $\nu$ is Possion's ratio. The maximum deflection, $w_{\max }$, at center of the plate is then,

$$
w_{\max }=\frac{p a^{4}}{64 D}
$$

The small deflection assumption is valid, when the maximum deflection is smaller than one fifth of the thickness. From this condition following constraint is derived:

$$
\frac{a}{h}<\left(\frac{64 E}{12 p\left(1-\nu^{2}\right)} \frac{1}{5}\right)^{0.25}
$$


The strain energy of bending, $V$, has the form [11]

$$
\begin{aligned}
V & =\frac{1}{2} \int_{0}^{2 \pi} \int_{0}^{a}\left(M_{r} \kappa_{r}+M_{t} \kappa_{t}\right) r \mathrm{~d} r \mathrm{~d} \theta \\
& =\frac{D}{2} \int_{0}^{2 \pi} \int_{0}^{a}\left[\left(\frac{\partial^{2} w}{\partial r^{2}}\right)^{2}+\frac{1}{r^{2}}\left(\frac{\partial w}{\partial r}\right)^{2}+\frac{2 \nu}{r} \frac{\partial^{2} w}{\partial r^{2}} \frac{\partial w}{\partial r}\right] r \mathrm{~d} r \mathrm{~d} \theta \\
& =\frac{32 \pi}{3} \frac{w_{\max }^{2}}{a^{2}} D .
\end{aligned}
$$

where $M_{r}$ and $M_{t}$ are the bending moments, and $\kappa_{r}$ and $\kappa_{t}$ are their curvatures. Hence, the strain energy release rate, $G$, is given by

$$
G=\frac{\mathrm{d} V}{\mathrm{~d} \pi a^{2}}=\frac{32 D w_{\max }^{2}}{a^{4}} .
$$

If the deflection is not small enough so that the small deflection assumption is not valid any more, the membrane strains at the mid-plane needs to be considered. Taking the radial displacement into account, the strain energy due to the stretching of the mid-plane, $V_{1}$, is given by $(\nu=0.3)$

$$
V_{1}=\frac{\pi E h^{2}}{1-\nu} \int_{0}^{a}\left(\varepsilon_{r}^{2}+\varepsilon_{t}^{2}+2 \nu \varepsilon_{r} \varepsilon_{t}\right) r \mathrm{~d} r .
$$

where $\varepsilon_{r}$ and $\varepsilon_{t}$ are the radial and the tangential strains. $\varepsilon_{r}$ and $\varepsilon_{t}$ are related respectively to the radial displacement, $u$, and the vertical deflection, $w$, by

$$
\varepsilon_{r}=\frac{\mathrm{d} u}{\mathrm{~d} r}+\frac{1}{2}\left(\frac{\mathrm{d} w}{\mathrm{~d} r}\right)^{2}
$$

and

$$
\varepsilon_{t}=\frac{u}{\mathrm{r}}
$$

The ansatz function for the radial displacement, $u$, may take the form ,

$$
u=r(a-r)\left(C_{1}+C_{2} r+C_{3} r^{2}+\cdots\right)
$$

where the coefficients of the terms are determined from the condition that the total potential energy of the plate is minimized in the state of static equilibrium. The total strain energy of a plate with large deflection consists of the contributions from the bending and membrane strains and is given by

$$
V+V_{1}=\frac{32 \pi}{3} \frac{w_{\max }^{2}}{a^{2}} D\left(1+0.244 \frac{w_{\max }^{2}}{h^{2}}\right)
$$


where the first two terms of radial displacements are taken. Then the strain energy release rate, $G_{0}$, due to extension of a crack (i.e. increasing the radius of the plate) can be obtained by

$$
G_{0}=\frac{\mathrm{d}(V+V 1)}{\mathrm{d} \pi a^{2}} .
$$

The maximum deflection under large deflection can be derived by applying the principle of virtual displacement and is given as

$$
w_{\max }=\frac{p a^{4}}{64 D} \frac{1}{1+0.488 \frac{w_{\max }^{2}}{h^{2}}} .
$$

\section{Thick plate}

If the blister cap is so thick that Equation 3 is not valid, the shear strain on any cross section of the cap needs to be considered. In this circumstance the analytical solution has a highly complicated form. For the sake of simplicity, a thick blister may be approximated as being embedded in an infinite elastic media. In this case, the maximum opening at the center of the cap, $\delta$, can be given in a simple form [12]

$$
\delta=\frac{8\left(1-\nu^{2}\right)}{\pi E} p a
$$

The stress intensity factor, $K_{\mathrm{I}}$, is given 12 ]

$$
K_{\mathrm{I}}=\frac{2}{\pi} p \sqrt{\pi a}
$$

In a linear elastic material, the strain energy release rate is related to the stress intensity factor as

$$
G=\frac{K_{\mathrm{I}}^{2}}{E}\left(1-\nu^{2}\right)=\frac{4 \pi p^{2} a\left(1-\nu^{2}\right)}{E} .
$$

In the present study plasticity is not included in the analytical investigation due to exceeding complexity of formulation, but instead, considered only in the FEA.

\section{Finite element model}

Fig. 3 shows a schematic drawing of the model geometry and the global mesh of the two-dimensional FEA model. The axi-symmetric plane model was created for the right half of the vertical cross section. The disk-shaped blister cap was defined by inserting a thin crack between the cap and the substrate. The commercial FEA code ABAQUS was employed for simulations [13]. Axi-symmetric quadratic solid element with reduced integration was used. Gas pressure was applied to the inner face of the blister cap.

The displacement components on the bottom face of the model were constrained assuming a semi-infinite substrate. 


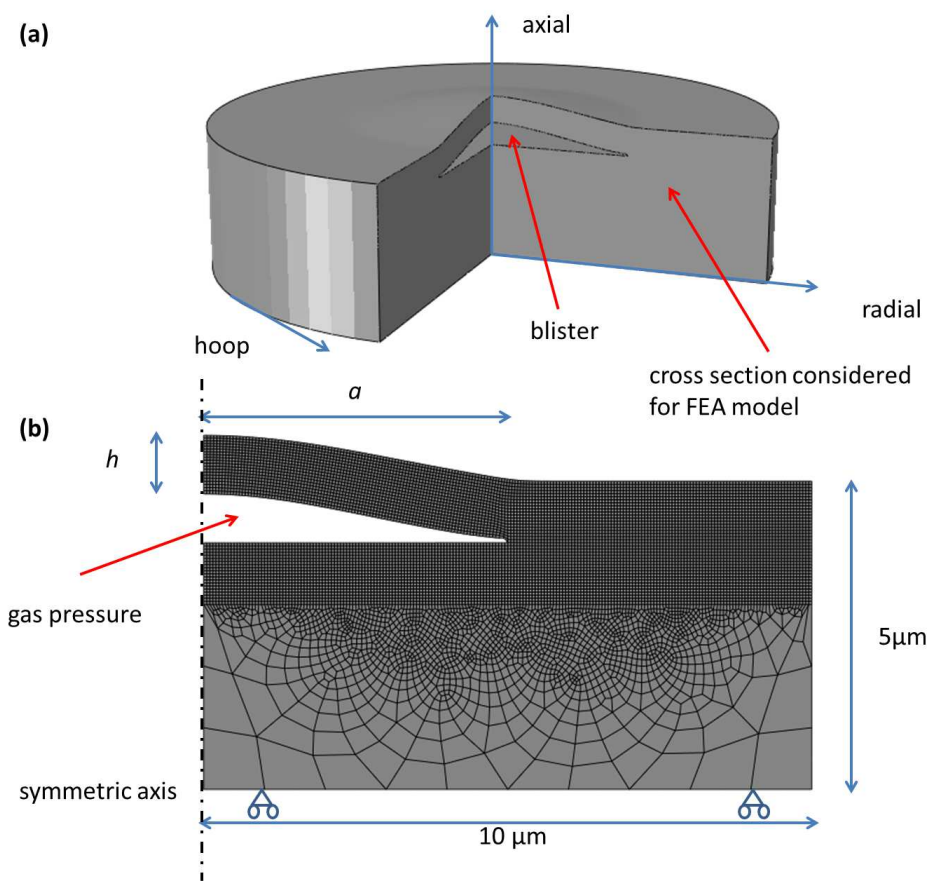

Fig. 3. (a) A schematic drawing of the model geometry. (b) Two-dimensional finite element model created for the right half of the vertical cross section. The mesh consists of axisymmetric elements considering rotational symmetry of the model.

\subsection{Material properties}

Tungsten exhibits fully isotropic elastic properties in a mono- as well as polycrystalline state. Thus, only two elastic constants are required for describing the linear elastic behavior of the tungsten grains in a blister on mesoscopic length scale. Literature data of elastic constants were used: $E=400 \mathrm{GPa}, \nu=0.3$.

The plastic behavior of undamaged metals is essentially determined by initial yield and work hardening. In this study, the Ramberg-Osgood deformation model was applied for elasto-plastic description. In onedimensional case, the Ramberg-Osgood model is expressed as

$$
\varepsilon=\frac{\sigma}{E}+\alpha \frac{\sigma_{0}}{E}\left(\frac{|\sigma|}{\sigma_{0}}\right)^{(n-1)},
$$

where $\sigma$ is the stress, $\varepsilon$ the strain, $\alpha$ the yield offset parameter, $\sigma_{0}$ the yield stress and $n$ is the hardening exponent. Assuming the $0.2 \%$ offset strain criterion for yield stress, the coefficient of the plastic term is further simplified as follows:

$$
\alpha \frac{\sigma_{0}}{E}=0.002
$$

Now, the plastic flow behavior is fully defined by two plastic parameters, namely, yield stress and hardening exponent. It is noted that the tensile stress-strain curves of tungsten single crystals can be qualitatively well described using the Ramberg-Osgood model [14]. Although yield stress generally plays an 
important role in plastic behavior, its impact on blister deformation can hardly be assessed in quantitative terms. This is because the yield stress of tungsten can be affected by the presence of highly populated hydrogen solutes while the in-situ measurement of yield stress during hydrogen implantation seems obviously difficult. Substantially reduced slip resistance of tungsten single crystals after hydrogen implantation was already observed [15].

In this work, a parametric investigation was carried out assuming a range of yield stress from 500 to $1000 \mathrm{MPa}$ which corresponds to the yield stress values of tungsten single crystals in different crystallographic orientations [14, 15]. Two different values of hardening exponent, 6 and 10, were assumed. In Fig. 4 the tensile stress-strain curves for three selected combinations of the plastic parameters are reproduced using the Ramberg-Osgood model showing the effect of each parameter.

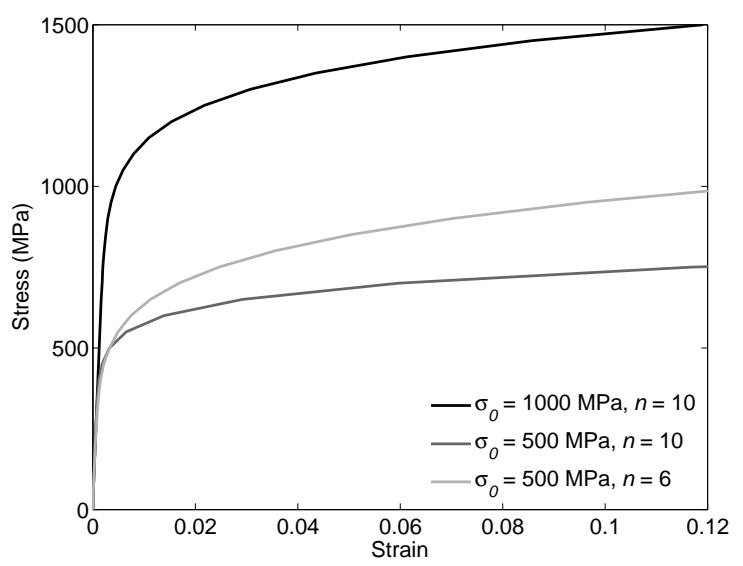

Fig. 4. Tensile stress-strain curves reproduced based on the Ramberg-Osgood deformation theory for three selected combinations of the plastic parameters.

\section{Results and discussion}

\subsection{Elastic analyzes}

In the elastic analysis the blister cap thickness was varied from 0.1 to $1 \mu \mathrm{m}$ while the radius was fixed at $5 \mu \mathrm{m}$. So, the aspect ratio $(a / h)$ was varied from 50 to 5 . The aspect ratio is chosen as the parameter in this work, since continuum mechanics is in itself scale-independent. Identical behavior will be predicted as long as the proportion of the model geometry (i.e. the aspect ratio) and the pressure-to-thickness ratio remain unchanged. Constant gas pressure of $100 \mathrm{MPa}$ was applied as a reference load, which corresponds to pressure values $(40-90 \mathrm{MPa})$ determined by the blister volume and the amount of released $\mathrm{D}_{2}$ molecules 16 .

In Fig. [5 the values of deflection of the cap at the uppermost position are plotted. Both the analytical and FEA predictions are presented and compared for the range of the aspect ratio. The analytical results were obtained separately from the thin plate theory assuming small and large deflections, respectively. In the same manner the FEA simulations were made separately with and without the assumption of geometrical non-linearity, respectively. Without the assumption of geometrical non-linearity, the original coordinates of nodes will be used in the FEA by assuming a small displacement, which will result in large errors when 
large deformation occurs [13]. As a result, the FEA simulation without the assumption of geometrical nonlinearity does not produce radial displacement at the mid-plane which accords with the assumption of small deflection in the analytical solution.

It is seen that the analytical and the numerical results agree well with each counterpart for the whole thickness range. In all cases the deflection (i.e. bulging) decreases monotonically with the increasing thickness, yet the predictions exhibit significant deviation between the small and the large deflection models, when the cap thickness is decreased below roughly $10 \%$ of the blister radius $(a / h>10)$. In the present case with the blister radius of $5 \mu \mathrm{m}$, the critical thickness would lie around $0.5 \mu \mathrm{m}$. The discrepancy is predicted both in the numerical and analytical cases in the same way. As the small deflection assumption is an approximation which neglects the membrane strains, the deformation can be overestimated for the cases in which the membrane strains cannot be neglected. This implies that geometrical non-linearity should be considered for modelling the large bulging (e.g. $a / h>10, p=100 \mathrm{MPa}$ ). In this regard, the criterion given in Equation 3 is worth evaluating. For the pressure of $100 \mathrm{MPa}$, the lower bound of the ratio, $a / h$, is estimated to be 8.3 for the small deflection assumption to be valid, and for $50 \mathrm{MPa}$, the aspect ratio should be smaller than 10. However, the thin plate assumption gives a lower bound of the aspect ratio. If the aspect ratio is smaller than 10, there is a deviation between the analytical and FEM solutions (see Fig. (5) comes from the fact that analytical solution under the thin plate assumption does not include the shear deformation. As the plate is thicker compared to the radius, the effect of the shear deformation cannot be neglected. The analytical solution with the thin plate assumption can therefore only be used if the aspect ratio is larger than 10. Considering the validity of both thin plate and small deflection assumptions, the analytical solution assuming small deflections can be used as a rough estimation only if the pressure is lower than $50 \mathrm{MPa}$.

Fig. 6 presents the strain energy release rate due to the growth of a blister as a function of the aspect ratio. As in Fig. 5, both the analytical and the FEA predictions are presented comparing the results of the small deflection models with those of the large deflection ones, respectively. The analytical data of energy release rate were estimated by means of the LEFM models given in Equation 5 for small deflection and Equation 11 for large deflection, respectively. In the FEA simulation of blister growth, the strain energy release rate is assessed by means of the standard LEFM method based on crack opening and virtual extension of crack tip. The strain energy release rate may be thought of as a measure of driving force for crack extension. The predictions show very similar trends as those of the cap deformation such that the energy release rate decreases as the thickness increases and that the estimates (analytical and numerical) of the small deflection approximation becomes considerably overestimated for the case of small thickness $(a / h$ $>10)$.

As far as the LEFM behavior of a blister is concerned, the FEA simulation with geometrical non-linearity seems to deliver the most accurate estimation. On the other hand, the validity of the analytical prediction with large deflection approximation for energy release rate seems to break down for small thickness $(a / h$ $>10)$. This may be due to the approximation of radial strain in Equation 6 . In the case of very large deflections, a further higher order term needs to be included as follows [17]

$$
\varepsilon_{r}=\frac{\mathrm{d} u}{\mathrm{~d} r}+\frac{1}{2}\left[\left(\frac{\mathrm{d} u}{\mathrm{~d} r}\right)^{2}+\left(\frac{\mathrm{d} w}{\mathrm{~d} r}\right)^{2}\right] .
$$

Noting that energy release rate decreases with increasing cap thickness, the lower bound of energy release 
rate can be estimated assuming a very thick blister cap using Equation 15. The fracture energy (twice the surface energy) of tungsten single crystals ranges roughly from 0.1 to $1.1 \mathrm{~mJ} / \mathrm{mm}^{2}$ at room temperature depending on the crystallographic orientation of cleavage surface [18]. In the present case, the lower bound value is estimated to be $1.4 \times 10^{-4} \mathrm{~mJ} / \mathrm{mm}^{2}$ which is three orders of magnitude smaller than the fracture energy of tungsten. This means that blisters situating at a deep position from the surface can enlarge itself only under extreme high pressure (at least several GPa).

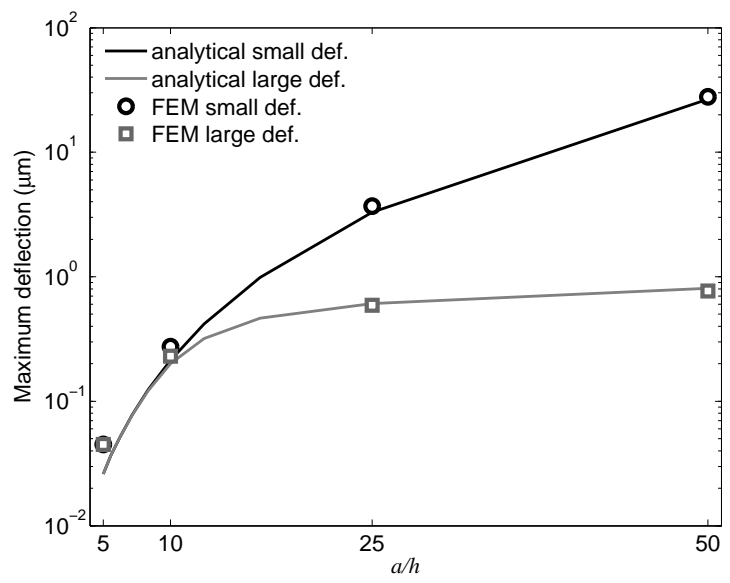

Fig. 5. Maximum bulge deflection of the cap under the gas pressure of $100 \mathrm{MPa}$ plotted as a function of the aspect ratio $a / h$ on a logarithmic scale $(h=1 \mu \mathrm{m})$. Analytical and numerical estimations are presented for small and large deflection assumption, respectively.

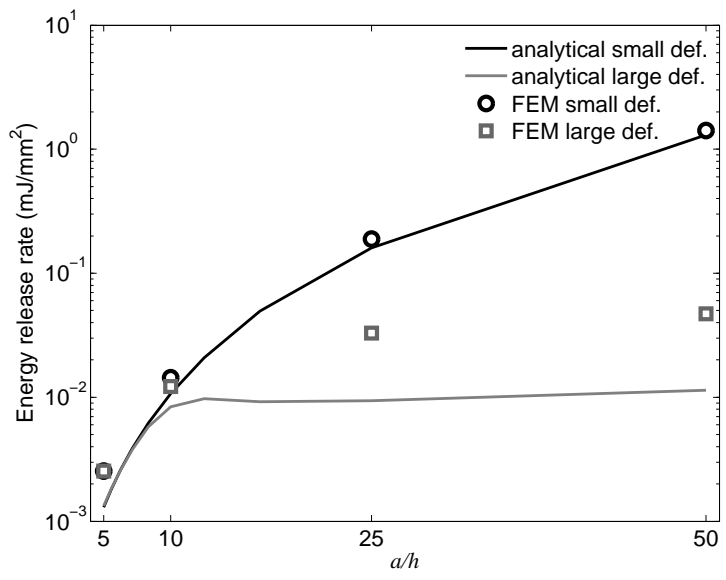

Fig. 6. Strain energy release rate at the blister cap periphery under the gas pressure of $100 \mathrm{MPa}$ plotted as a function of the aspect ratio $a / h$ on a logarithmic scale $(h=1 \mu \mathrm{m})$. Analytical and numerical estimations are presented for small and large deflection assumption, respectively.

\subsection{Elasto-plastic calculation}

In this section, parametric simulations were carried out where internal pressure was varied from 50 to $200 \mathrm{MPa}$ while the radius of the blister was varied from 2 to $5 \mu \mathrm{m}$. Two different cap thicknesses $(1 \mu \mathrm{m}$, 
$0.5 \mu \mathrm{m})$ were assumed.

Fig. 7] shows the computed plastic strain field produced by the gas pressure of $100 \mathrm{MPa}$ as an example. Plotted is the equivalent plastic strain, $\varepsilon_{\mathrm{eq}}^{\mathrm{pl}}$, defined $\mathrm{as}^{1}$ :

$$
\varepsilon_{\mathrm{eq}}^{\mathrm{pl}}=\sqrt{\frac{2}{3} \varepsilon_{i j}^{\mathrm{pl}} \varepsilon_{i j}^{\mathrm{pl}}} .
$$

where $\varepsilon_{i j}^{\mathrm{pl}}$ is the plastic strain tensor component.

The plastic strain is locally concentrated at the cap apex and the blister periphery which acts like a crack tip. The strong localization of plastic strain indicates that these two areas would be the most critical sites being vulnerable to damage and rupture. In the following we examine the plastic deformation behavior of the blister focusing on these two sites.

In Fig. 8, the equivalent plastic strains at the cap apex (a) and the crack tip (b) are plotted as a function of yield stress for different aspect ratios. It is seen that the plastic deformation at the crack tip is much more significant than at the cap apex. The plastic strain increases with increasing the aspect ratio. When the aspect ratio increases above a certain limit for the given thickness, the blister exhibits an accelerated plastic deformation at lower yield stress. In the present case, this limit is reached roughly at $a / h=5$ at which the blister undergoes abruptly enhanced bulging deformation for the yield stress lower than $700 \mathrm{MPa}$. When the yield stress is lower than $500 \mathrm{MPa}$, then the blister would be likely to fail at the periphery (crack tip) owing to plastic instability. This feature was already discussed in the previously published companion paper [10].

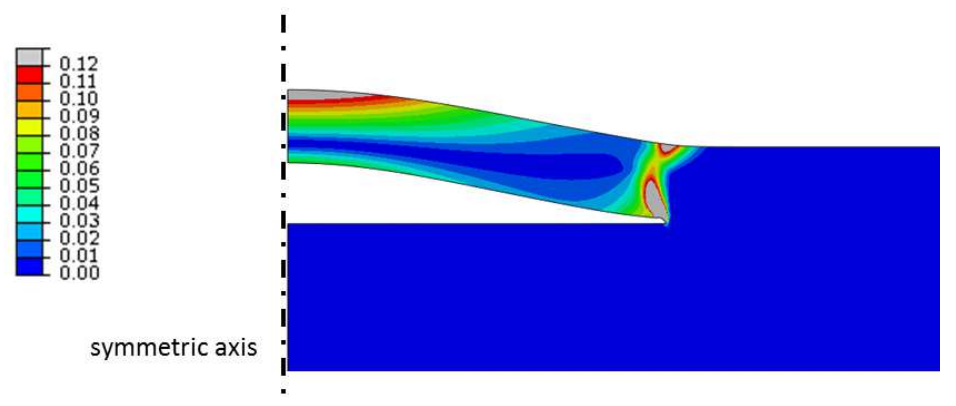

Fig. 7. Equivalent plastic strain field around a tungsten blister produced by the gas pressure of $100 \mathrm{MPa} .\left(\sigma_{0}=500 \mathrm{MPa}, a / h\right.$ $=5$ and $n=10$.)

Next, we examine the fracture mechanical behavior of the blister. The driving force of crack extension (and thus blister growth) in a plastic solid is described with a dedicated scalar-valued parameter, the $J$ integral. The $J$-integral is the plastic counterpart of the strain energy release rate $G$ of a sharp crack in an elastic solid. Therefore, the $J$-integral can be used as a criterion for crack extension in analogy to $G$. The critical value of the $J$-integral, $J_{\mathrm{c}}$, is the specific energy required for extending a crack of unit area and thus a material property representing the fracture toughness of the material. In the linear elastic limit the value of the $J$-integral converges to that of $G$.

\footnotetext{
${ }^{1}$ Since deformation plasticity is applied in the simulations and all the deformation is classified to plastic deformation in ABAQUS.
} 


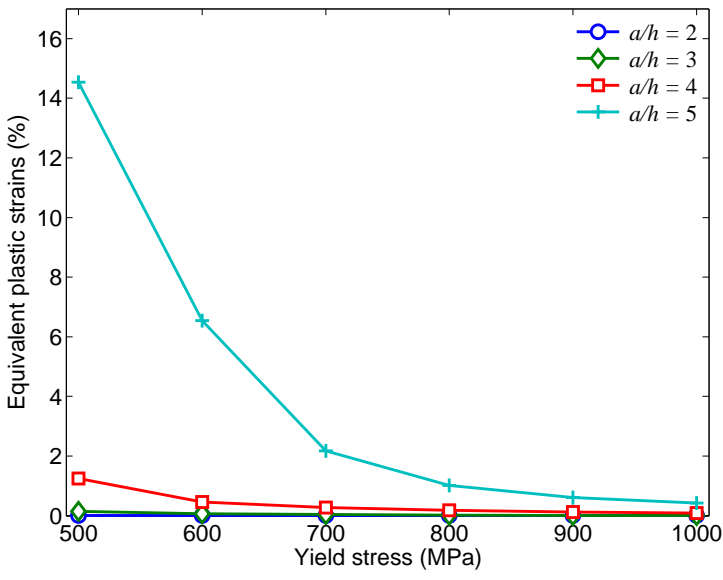

(a) Equivalent plastic strains at the dome apex

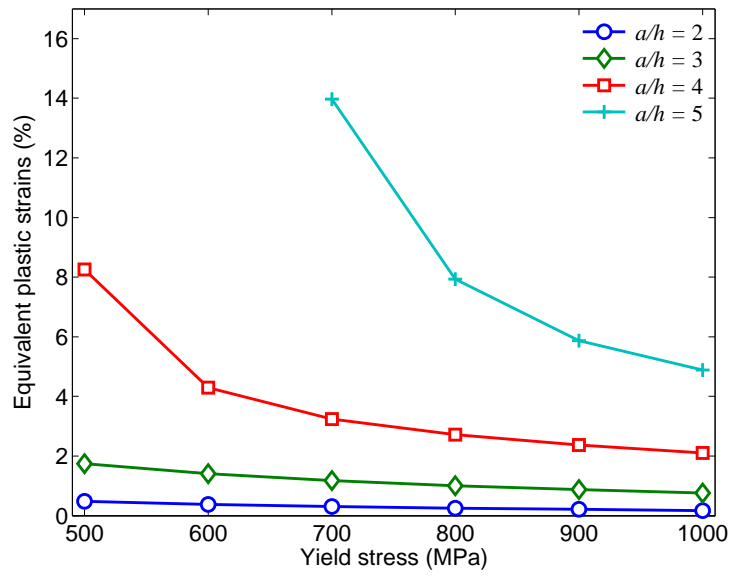

(b) Equivalent plastic strains at the crack tip

Fig. 8. Equivalent plastic strain at the cap apex (a) and the crack tip (b) plotted as a function of yield stress for different aspect ratios. $(h=1 \mu \mathrm{m}, p=100 \mathrm{MPa}$ and $n=10$.)

In Fig. 9, the $J$-integral values at the crack tip (periphery) are plotted as a function of yield stress for different aspect ratios. It is seen that the $J$-integral behaves in a similar fashion as the plastic strain. For the given cap thickness $(1 \mu \mathrm{m})$, the $J$-integral of the smaller blisters $(a / h<4)$ remains almost constant and fairly low for the whole range of considered yield stress being actually equal to the values of linear elastic limit, that is, $G$ values. For this range of blister dimension $(a / h<4)$ the estimated $J$-integral values are smaller than $0.005 \mathrm{~mJ} / \mathrm{mm}^{2}$. The larger blister with higher aspect ratio $(a / h=5)$ shows a significant increase of the $J$-integral at lower yield stress below $700 \mathrm{MPa}$. The $J$-integral of this slender blister increases up to $0.025 \mathrm{~mJ} / \mathrm{mm}^{2}$ at yield stress of $500 \mathrm{MPa}$. From the comparison of the estimated $J$-integral values with the values of fracture energy of tungsten, it is obvious that the driving force for crack extension (i.e. blister growth) is not strong enough for the blisters with the aspect ratio between $2<a / h<5$ at the given gas pressure of $100 \mathrm{MPa}$.

Then, we discuss the deformation behavior of blister under changing gas pressure. Fig. 10 shows the equivalent plastic strain at the cap apex and the crack tip plotted as a function of yield stress $(n=10)$ and for four different gas pressures. Here, the geometry was fixed at the aspect ratio of $a / h=3$. Under the varying pressure the blister exhibits a very analogous response as that of the blister with changing aspect ratio, that is, the blister abruptly begins to plastically bulge out at some stage of gas pressure, provided that the yield stress is sufficiently low $(<600 \mathrm{MPa})$. In the current case $(a / h=3)$ this critical pressure is $200 \mathrm{MPa}$. At this pressure the periphery (crack tip) of the blister seems to undergo plastic instability at lower yield stress $(<600 \mathrm{MPa})$.

In Fig. 11 the $J$-integral values at the crack tip of the same blister are plotted as a function of yield stress $(n=10)$ for four different gas pressures. The $J$-integral response exhibits a similar trend as that of equivalent plastic strain. At the current aspect ratio $(a / h=3)$ the critical pressure is $200 \mathrm{MPa}$. The salient increase of the $J$-integral occurs only when the yield stress is low enough $(<600 \mathrm{MPa})$.

The abrupt and rapid increase of plastic deformation and the $J$-integral at a certain critical stage of aspect ratio, yield stress and gas pressure is one of the most prominent characteristics in the mechanical behavior of a blister. This feature can be understood with the help of stress fields. In Fig. 12, two pairs of 


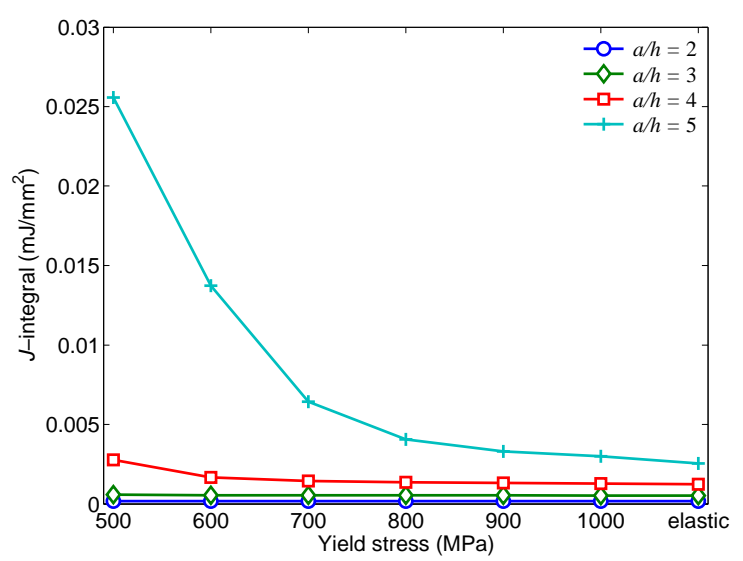

Fig. 9. J-integral values at the blister periphery (crack tip) plotted as a function of yield stress for different aspect ratios. $(h$ $=1 \mu \mathrm{m}, p=100 \mathrm{MPa}$ and $n=10$.)

stress field are presented where the von Mises stress field produced before and after the critical stage are compared for each loading condition, respectively. The von Mises stress is a scalar-valued function of the second stress invariant and can be interpreted as a measure of yield criterion.

The yield stress was fixed at $500 \mathrm{MPa}$. In the first comparison, the pressure was fixed at $100 \mathrm{MPa}$ and the aspect ratio $(a / h)$ was increased from 3 to 4 . It is seen that the stress field develops abruptly and strongly throughout the blister cap. The von Mises stress exceeds the assumed yield stress (500 MPa) in the whole cap indicating that the plastic zone sweeps the whole cap region. This circumstance might lead to a plastic instability at higher pressure. It is noted that the stress level before the critical stage is mostly very low, although stress begins to concentrate at the crack tip.

The same trend is observed in the second comparison where the pressure was increased from $150 \mathrm{MPa}$ to $200 \mathrm{MPa}$ while the aspect ratio was fixed at $a / h=3$.

In Fig. 13 the $J$-integral values of the blister with $a / h=5$ are plotted as a function of yield stress for two different values of work hardening exponent, $n=6$ and 10. The applied gas pressure is $100 \mathrm{MPa}$. The value of $\mathrm{n}$ is reciprocally proportional to the work hardenability. Hence, the larger $n$ will produce more enhanced plastic strains and thus stronger $J$-integral than the smaller $n$. The predicted trend of the $J$-integral in Fig. 13 exactly reproduces this feature. The effect of $n$ diminishes rapidly as yield stress increases, since the deformation begins to be dominated by elasticity. 


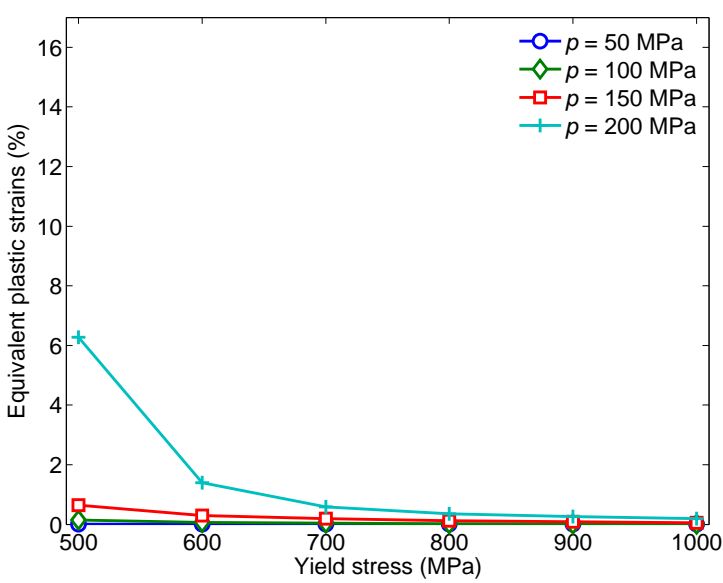

(a) Equivalent plastic strains at the dome apex

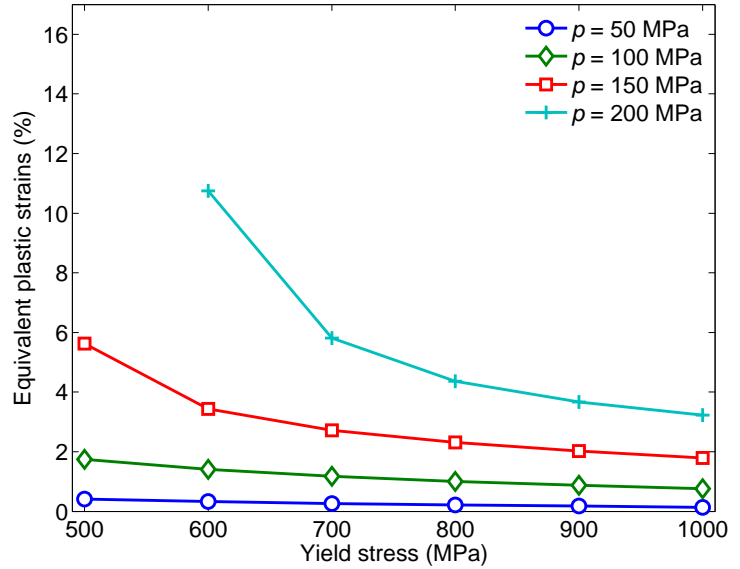

(b) Equivalent plastic strains at the crack tip

Fig. 10. Equivalent plastic strains at two critical positions (i.e. cap apex, crack tip) of the blister plotted as a function of yield stress $(n=10)$. The data were computed for four different gas pressures and a fixed aspect ratio $(a / h=3)$.

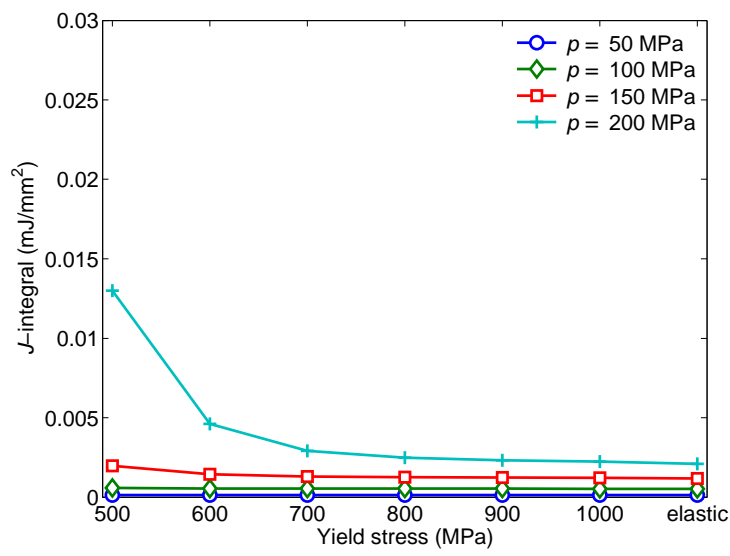

Fig. 11. The $J$-integral at the crack tip of the blister with the aspect ratio of $a / h=3$ as a function of yield stress $(n=10)$. The data were computed for four different gas pressures. 

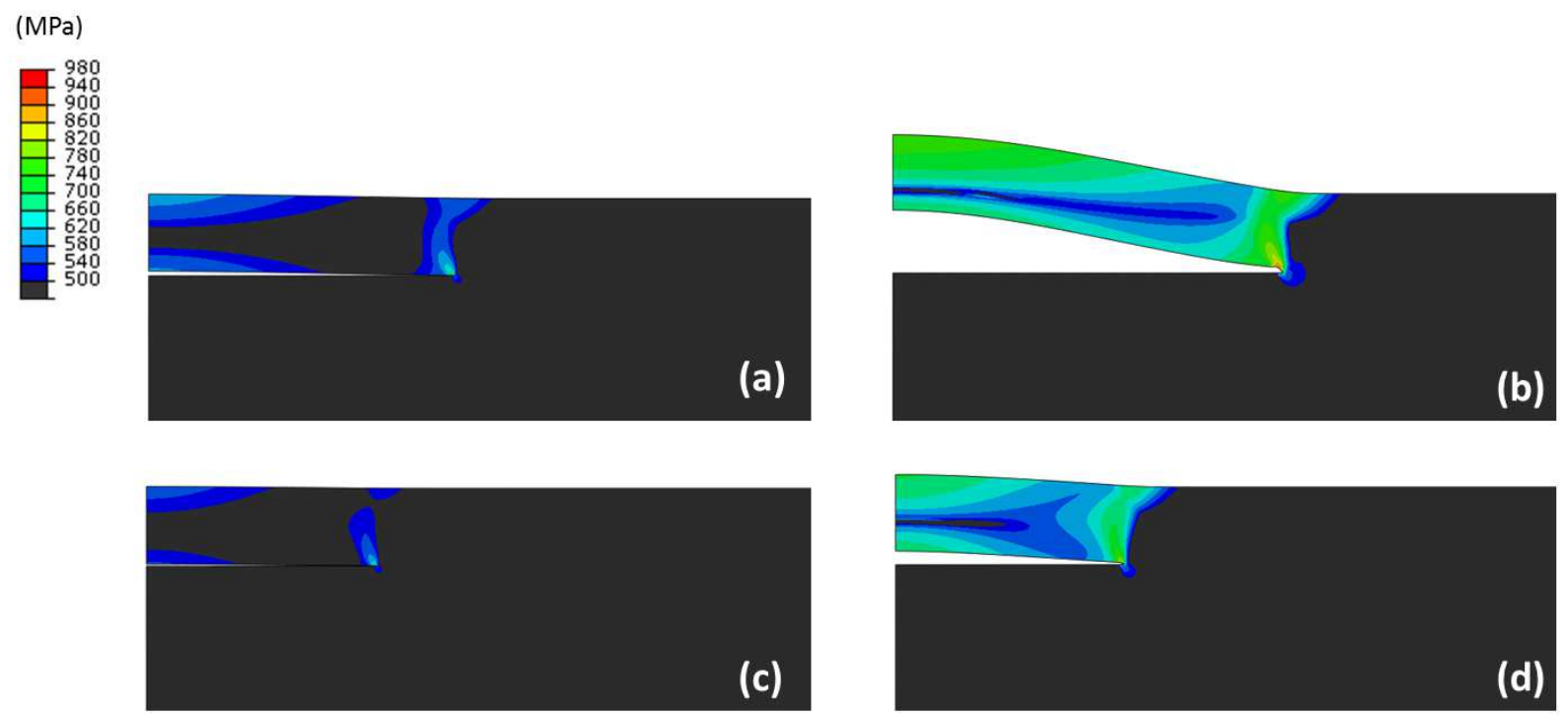

Fig. 12. Von Mises stress fields, $\sigma_{0}=500 \mathrm{MPa},(\mathrm{a}): a / h=4, p=100 \mathrm{MPa},(\mathrm{b}): a / h=5, p=100 \mathrm{MPa},(\mathrm{c}): a / h=3, p=$ $150 \mathrm{MPa},(\mathrm{d}): a / h=3, p=200 \mathrm{MPa}$.

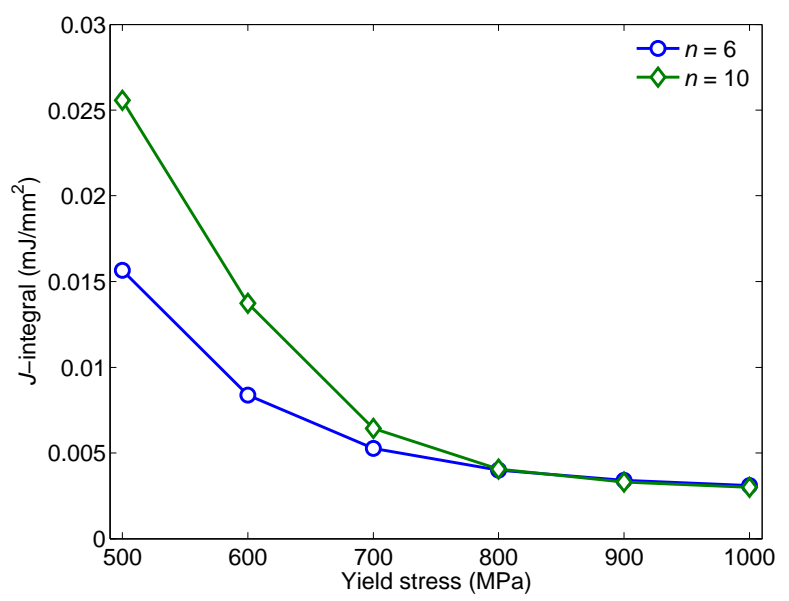

Fig. 13. $J$-integrals as a function of yields stresses for different hardening parameters. $(a / h=5$ and $p=100 \mathrm{MPa}$. 


\section{Conclusions}

In this article we presented a generic theoretical framework to describe the bulging deformation of blisters and to estimate the mechanical driving force of blister growth. As reference material tungsten was considered with assumed variation of yield stress and work hardening exponent. The validity of the analytical formulations based on the elastic plate theories was evaluated with the help of FEA. The plastic strains and $J$-integral values at the boundary edge were assessed by means of FEA. Extensive parametric studies were performed for a range of blister aspect ratio $(1<a / h<5)$, gas pressure $(50-200 \mathrm{MPa})$, yield stress $(500-1000 \mathrm{MPa})$ and hardening exponent $(6-10)$. Following conclusions were reached:

1. The analytical solutions of thin plate theory can be applied, provided the bulging deformation is moderate. This condition is met, when the gas pressure is low (roughly $50 \mathrm{MPa}$ ). For better accuracy general solutions with large deflection correction should be used.

2. The analytically estimated elastic strain energy release rate exhibits a similar trend as the elastic deformation.

3. In the elasto-plastic case, the $J$-integral at the blister boundary edge showed the same trend as that of the plastic strain in the blister cap: it increased with decreasing yield stress, increasing gas pressure and increasing aspect ratio.

4. Both the plastic strain in the blister and the $J$-integral at the blister edge exhibit an abrupt and strong increase at certain critical stages of pressure, geometry (aspect ratio) and yield stress. Assuming the yield stress is $500 \mathrm{MPa}$, the critical pressure is $200 \mathrm{MPa}$ at the aspect ratio of 3 , while at the pressure of $100 \mathrm{MPa}$, the critical aspect ratio is 5 . This plastic and fracture instability is one of the most characteristic features of the blistering mechanics.

\section{References}

[1] M. Balden, S. Lindig, A. Manhard, J.-H. You, J. Nucl. Mater. 414 (2011) 69-72.

[2] L. Gao, U. von Toussaint, W. Jacob, M. Balden, A. Manhard, Nucl. Fusion 54 (2014) 122003.

[3] M. H. J. 't Hoen, M. Balden, A. Manhard, M. Mayer, S. Elgeti, A. W. Kleyn, P. A. Z. van Emmichoven, Nucl. Fusion 54 (2014) 083014.

[4] R. A. Causey, J. Nucl. Mater. 300 (2002) 91-117.

[5] M. Risch, J. Roth, B. Scherzer, J. Nucl. Mater. 82 (1979) 220-226.

[6] W. Han, J. Yu, J. Appl. Phys. 89 (2001) 6551.

[7] J. W. Hong, S. W. Cheong, J. Appl. Phys. 100 (2006) 094322.

[8] Z. Cao, P. Wang, W. Gao, L. Tao, J. Suk, R. Ruoff, D. Akinwande, R. Huang, K. Liechti, Carbon 69 (2014) $390-400$.

[9] N. Enomoto, S. Muto, T. Tanabe, J. Davis, A. Haasz, J. Nucl. Mater. 385 (2009) 606-614.

[10] J.-H. You, J. Nucl. Mater. 437 (2013) 24-28.

[11] S. Timoshenko, S. Woinowsky-Krieger, Theory of plates and shells, New York : McGraw-Hill, 2nd ed edition, 1959.

[12] H. Tada, P. C. Paris, G. R. Irwin, The Stress Analysis of Cracks Handbook, American Society of Mechanical Engineers Press, third edition, 2001.

[13] Dassault Systémes Simulia Corp., Providence, RI, USA, 2012 (2012).

[14] P. Beardmore, D. Hull, J. Less-Common Met. 9 (1965) 168-180.

[15] W. Z. Yao, P. Wang, A. Manhard, C. E. Krill III, J.-H. You, Materials Science and Engineering: A 559 (2013) $467-473$.

[16] M. Balden, S. Lindig, A. Manhard, V. K. Alimov, O. V. Ogorodnikova, J. Roth, 13th International Workshop on PlasmaFacing Materials and Components for Fusion Applications and 1st International Conference on Fusion Energy Materials Science, Rosenheim, Germany, 2011.

[17] E. Reissner, Proc. of Symposia in Appl. Math. 1 (1949) 213 - 219.

[18] J. Riedle, P. Gumbsch, H. F. Fischmeister, Phys. Rev. Lett. 76 (1996) 3594. 